\title{
The Genetic Basis of Natural Variation in Drosophila melanogaster Immune Defense against Enterococcus faecalis
}

\author{
Joanne R Chapman ${ }^{1, *}$, Maureen A Dowell ${ }^{1,2} \mathbb{D}$, Rosanna Chan ${ }^{1}$ and Robert L Unckless ${ }^{1}$ (D) \\ 1 Department of Molecular Biosciences, University of Kansas, Lawrence, Kansas, KS 66045, USA; \\ maureen_dowell@brown.edu (M.A.D.); rosannachan01@gmail.com (R.C.); unckless@ku.edu (R.L.U.) \\ 2 Department of Molecular Biology, Cell Biology \& Biochemistry, Brown University, Providence, \\ Rhode, RI 02912, USA \\ * Correspondence: joanne.chapman@ku.edu; Tel.: +1-785-864-1679
}

Received: 29 January 2020; Accepted: 18 February 2020; Published: 22 February 2020

\begin{abstract}
Dissecting the genetic basis of natural variation in disease response in hosts provides insights into the coevolutionary dynamics of host-pathogen interactions. Here, a genome-wide association study of Drosophila melanogaster survival after infection with the Gram-positive entomopathogenic bacterium Enterococcus faecalis is reported. There was considerable variation in defense against $E$. faecalis infection among inbred lines of the Drosophila Genetics Reference Panel. We identified single nucleotide polymorphisms associated with six genes with a significant $\left(p<10^{-08}\right.$, corresponding to a false discovery rate of $2.4 \%$ ) association with survival, none of which were canonical immune genes. To validate the role of these genes in immune defense, their expression was knocked-down using RNAi and survival of infected hosts was followed, which confirmed a role for the genes krishah and $S 6 \mathrm{k}$ in immune defense. We further identified a putative role for the Bomanin gene BomBc1 (also known as IM23), in E. faecalis infection response. This study adds to the growing set of association studies for infection in Drosophila melanogaster and suggests that the genetic causes of variation in immune defense differ for different pathogens.
\end{abstract}

Keywords: genome-wide association study (GWAS); innate immunity; immune response; systemic infection; Toll pathway; gram-positive bacteria

\section{Introduction}

Pathogenic microorganisms, those that cause increased morbidity and/or mortality in infected individuals, can pose serious threats to the fitness of infected individual hosts and the long-term survival of host populations, and even species [1,2]. In response to the challenges posed by pathogens, most hosts have developed a complex series of defense mechanisms, collectively known as the immune system. Yet, mounting an immune response to pathogens is costly in terms of both energy expenditure and resource allocation [3,4]. For this reason, selection should act on hosts to tolerate or resist pathogens based on the relative costs of harboring or clearing an infection. Furthermore, within host populations there tends to be genetic variation in immune defense, such that individuals vary in their response to specific pathogens. This variation in immune defense is not simply restricted to canonical immunity genes, but also to those genes involved in life-history, behavioral and physiological traits that influence the outcome of host-pathogen interactions [5]. Continual host-pathogen coevolution likely promotes maintenance of immune diversity in populations $[6,7]$. Understanding the genetic underpinnings of natural variation for immunity within a population or species therefore provides insights into the specific genes and immune pathways involved in the host immune response to a particular pathogen. 
Genome-wide association studies (GWAS) test for statistical associations between genotype and phenotype at each variable site in the genome, and theoretically have the power to detect alleles with only modest effects on phenotype [8]. One perceived benefit of GWAS is that it allows for unbiased detection of unknown and unexpected genotypic associations because the whole genome is interrogated simultaneously. While this method has been used to examine the genetic basis of immunity in Drosophila melanogaster previously e.g., [9-13], no study to date has used GWAS to examine Drosophila defense against Enterococcus faecalis.

E. faecalis is a Gram-positive bacterium with lysine-type peptidoglycan that readily infects insect and mammalian hosts [14], where it lowers individual survival [14,15]. E. faecalis occurs naturally in Drosophila populations, most likely at low prevalence [16,17]. Importantly, E. faecalis has been isolated from the hemolymph of wild D. melanogaster, and challenge with this bacterium at a relatively low dose causes intermediate levels of mortality in laboratory reared flies $[17,18]$. Thus, E. faecalis is not solely a gut commensal of $D$. melanogaster but rather can cause systemic pathogenic infections. As such, it is likely that there is selection for resistance and/or tolerance in D. melanogaster. Indeed, Toll-pathway induced antimicrobial peptides are upregulated in response to E. faecalis infection and play a key role in controlling the infection [19-21]. Recently, a new group of immune peptides, the Bomanins, were shown to play an important role in controlling Gram-positive infections such as E. faecalis in $D$. melanogaster $[15,22,23]$.

Here, we use a genome-wide association study to identify genetic variation involved in $D$. melanogaster immune defense against infection with E. faecalis. Subsequently, expression of putative response genes was knocked down in vivo using RNAi and survival after infection was monitored. This allowed us to confirm the role of two genes involved in D. melanogaster survival after E. faecalis infection that have not previously been recognized as playing a role in immunity to this pathogen. In addition, we dissected the role that Bomanins play in regulating response to E. faecalis infection and showed BomBc1 may play a specific role in enhancing Drosophila survival when infected with this pathogen.

\section{Materials and Methods}

\subsection{Drosophila and Bacterial Stocks}

The Drosophila Genetic Reference Panel (DGRP) population was used in this study. The DGRP is a North American (Raleigh, NC, USA) set of inbred lines that was developed for analysis of quantitative traits [24]. Full genome sequences are available for every line $(n=205)$ [25]. Flies were housed at $22^{\circ} \mathrm{C}$ on a 12-h day/night cycle in vials containing standard Drosophila cornmeal-molasses-yeast food.

The E. faecalis strain used in this study was originally isolated from the hemolymph of wild-caught D. melanogaster [17]. One week before the start of the experiment, the bacterium was revived from a glycerol stock maintained at $-80^{\circ} \mathrm{C}$ by streaking out on a Lysogeny Agar plate (LA, Difco, Detroit, MI, USA) and incubating the plate at $37^{\circ} \mathrm{C}$ overnight. This plate was then stored at $4{ }^{\circ} \mathrm{C}$. The evening before infections were to be performed, a single colony was aseptically picked from the LA plate and placed in $2 \mathrm{~mL}$ of sterile Lysogeny broth (LB, Difco, Detroit, MI, USA) at $37^{\circ} \mathrm{C}$ with shaking (250 rpm) overnight. The next day, the bacterial suspension was spun down ( $1 \mathrm{~min}$ at 13,000 rpm) and resuspended in $\mathrm{LB}$ to a final $\mathrm{OD}_{600}$ of $1.5 \pm 0.02$, which represents a final bacterial concentration of $\sim 1.3 \times 10^{9} \mathrm{CFU} / \mathrm{mL}$.

\subsection{Genome-Wide Association Study}

All but three DGRP lines (DGRP_21, DGRP_49, DGRP_325) were included in this study, resulting in an initial dataset of 202 DGRP lines in the GWAS analysis. Four infectors (JRC, MAD, RC, RLU) aimed to each infect at least 10 individuals per line. Adult males were infected 4-7 days post-eclosion, via piercing of the thorax with a $\varnothing 0.1 \mathrm{~mm}$ pin that had been dipped in the bacterial suspension described above. We limited all our infection studies to males because female flies can exhibit different immune 
responses depending on mating status and reproductive investment [26-28]. We used 4-7-day-old adult flies because the fat body (the main immune tissue in Drosophila) is not fully mature and fixed for the first three days of the adult stage [29] and because flies undergo immunosenescence as they age [30]. Four to seven days, therefore, represents a period of life in which expression of immune genes should be optimal. Other flies were pricked with sterile LB to serve as uninfected controls (mean \pm s.d. $=15.95$ \pm 5.58 per line). After treatment, $4-10$ males from a given line (mean \pm s.d. $=9.93 \pm 0.45$ ) were placed in a vial containing cornmeal-molasses-yeast food, with each vial containing flies subjected to a single treatment by a single infector. Vials were then stored in an incubator at $22{ }^{\circ} \mathrm{C}$ on a $12 \mathrm{~h}$ day/night cycle. The number of surviving individuals was recorded five days after treatment, which is a reasonable proxy of infection outcome because numerous Drosophila infection studies have shown that flies tend to either die of infection within $48 \mathrm{~h}$ or survive beyond seven days, e.g., [22,31,32]. We found that DGRP line DGRP_321 was an extreme outlier in that most individuals died shortly after infection. This line has also been previously identified as having abnormally lowered immune functioning [13]. As such, we excluded DGRP_321 and conducted the GWAS analysis on the remaining 201 lines.

To determine whether E. faecalis survival is associated with a specific genetic variant in the DGRP, a generalized linear mixed model with a binomial distribution and a logit link was fitted to the survival data using Equation (1):

$$
\text { Proportion alive } \sim \text { Date + Infector + Wolbachia status + (1| Line)/variant, }
$$

where (1|Line)/variant refers to DGRP fly line nested within genetic variant (random effect), variant is the specific variant (A, C, T, or G for SNPs, longer sequence strings for insertions and deletions) at a given genomic position (fixed effect), Date is the day infections were conducted (fixed effect), Infector is the identity of the person performing infections for that vial (fixed effect) and Wolbachia status refers to whether the line is systemically infected with the intracellular bacterium Wolbachia pipientis (fixed effect). This test was performed for every variable position in the genome that met the following criteria: A) the site was biallelic amongst the phenotyped lines and B) the minor allele count was greater than five. This led to a total of $n=2,383,736$ independent tests.

\subsection{RNAi Knock-Down of Candidate Genes}

An initial list of variants putatively associated with E. faecalis survival in the DGRP was generated by applying a $p$-value cut-off of $p<10^{-7}$, corresponding to a false discovery rate of $23.8 \%$. To further winnow the list of variants to validate, we then applied a second, more conservative $p$-value cut-off of $p$ $<10^{-8}$ to reduce the false discovery rate to $2.38 \%$ and therefore retain only those variants with a strong association with survival after E. faecalis infection. Genes associated with variants significant at the $p<$ $10^{-8}$ level are hereafter termed genes of interest (GOIs). We chose to further dissect the role of a subset of these genes, as well as genes physically close to these candidate genes (which we termed neighbor genes), by knocking down their expression in-vivo using RNAi and following survival after infection with E. faecalis in comparison to 'empty cassette' controls (see below). The neighbor gene approach was chosen to control for the possibility that knocking down random genes produced a change in immune phenotype. To this end, we used flies created by the Transgenic RNAi Programme (TRiP) [33] which we obtained from the Bloomington Drosophila Stock Center. TRiP uses a Gal4-UAS system to drive down expression of the targeted gene when crossed to a driver line. We used two different driver lines, one (Act5C) targets knockdown across the whole body, the other (C564), targets the fat body. We used the fat body driver in cases where whole body knock-down of a gene was lethal, because the fat body is the major immune-related tissue in Drosophila, analogous to the mammalian liver [34,35]. To obtain knockdown F1 individuals, RNAi TRiP males were crossed to virgin females from the driver lines Act5C or C564, per gene of interest and 'empty cassette' controls (isogenic to the knockdown lines except for the specific hairpin-containing transgene) (Figure S1). F1 offspring from these crosses were collected onto fresh food 1-2 day-post-eclosion for subsequent infection (Figure S1). A balancer 
chromosome was present in some of the TRiP lines, in these cases we only infected FI progeny with the wild-type phenotype, because these individuals harbor the target RNAi transgene.

\subsection{E. faecalis Infection of RNAi Lines with Knock-Down of Candidate Genes}

As with the GWAS analysis, we infected 4-7-day-old males, in this case, F1 offspring from the crosses described above, with E. faecalis at a standardized concentration of $\mathrm{OD}_{600}=1.5 \pm 0.02$ and counted the number of survivors after five days. However, for these experiments, only two people infected flies (JRC and MAD). Three types of comparisons were made. First, comparisons were made between lines with identical genetic backgrounds except for the knock-down of one specific gene (target vs 'empty cassette' comparisons, where the target is either the GOI or neighbor gene, see below). Second, comparisons were made between knockdowns of the candidate GOI and a neighbor gene, being the closest genomic neighbor gene that was not itself identified in the GWAS (GOI vs neighbor comparisons). Third, lines were infected with either E. faecalis or sterile LB (infected vs sterile prick controls, data not shown). In total, we aimed to infect a minimum of 100 males per line (target/empty cassette per GOI/neighbor) with $E$. faecalis and a minimum of 30 males per line (target/empty cassette per GOI/neighbor) with LB (see Table S2), resulting in a minimum of 260 treated flies per experiment.

To determine whether GOI lines (i.e., those with expression of the candidate gene knocked-down by RNAi) had different survival than control lines without that gene knocked down, a generalized linear model with a binomial distribution and a logit link was fitted to the survival data. Depending on the number of infectors, we used Equation (2) (one infector) or Equation (3) (two infectors):

$$
\text { Proportion alive } \sim \text { Line }+ \text { Date, }
$$

$$
\text { Proportion alive } \sim \text { Line + Infector/Date, }
$$

where Line is the identity of the line (target or control) and Date is the date infections were performed. Infector/Date is date nested within infector. Equation (3) was only required for the analysis of neighbor gene CG42272, because both JRC and MAD infected flies for this line (note that only a single person infected on any given date). For all other genes, Equation (2) was fitted because a single person performed all infections. Wolbachia status was not required because Wolbachia is maternally transmitted and the two driver lines (Act5C and C564) are Wolbachia free. To visualize the differences in survival between target RNAi knockdown genes (GOI/neighbor) and empty cassette controls, we fitted a model using Equation (2) or Equation (3) as appropriate, then a second model without the Line effect. We used the residuals from this second model as the response (y) variable and plotted this against the Line effect from the full (Equation (2)/Equation (3)) model as the predictor (x) variable.

\subsection{Effects of Knocking Down Specific Bomanin Genes on E. faecalis Survival}

We additionally investigated the effect of transgenically reducing expression of four Bomanin genes (BomS1, BomBc1, BomT1 and BomS4) by knocking down expression, again using TRiP line males crossed to C564 females and infecting F1 males. Comparisons were made between specific Bom-individuals and control individuals containing the TRiP empty cassette, infected with E. faecalis at the standard dose $\left(\mathrm{OD}_{600}=1.5 \pm 0.02\right)$ or Todd-Hewitt $(\mathrm{TH})$ broth as a sterile control. TH was used in this experiment because it was found that E. faecalis grows better in this media. The use of LB or TH as growth medium and sterile prick control had no effect on survival of infected or control flies (data not shown). Five-day survival was recorded. Subsequently, we increased the dose to $\left(\mathrm{OD}_{600}=2.4\right.$ $\pm 0.02)$ to increase mortality rates, and again recorded survival after five days. All infections were conducted by JRC for this experiment.

To determine whether knock-down of specific Bomanin genes by RNAi had an effect on fly survival, a generalized linear model with a binomial distribution and a logit link was fitted to the survival data using Equation (2). In this case, Line was the identity of the Bomanin knock-down or control line. 


\subsection{Statistical Analyses}

All statistical analyses were performed using R v 3.4.1 [36]. Generalized models were fitted using the glmer function in the lme4 package [37] and graphics were plotted using ggplot2 [38] and corrplot [39].

\section{Results}

\subsection{Variation in Immunity to E. faecalis Infection in the DGRP}

In total, 27-65 males were infected with E. faecalis per line (mean \pm s.d. $=46.32 \pm 6.02$, Figure S2a). There was a more than three-fold difference in raw survivorship across the DGPR lines in their ability to survive infection for five days (Figure S2b). The five lines with lowest survival across infectors and days (DGRP_75, DGRP_176, DGRP_177, DGRP_313, DGRP_492) all had an average survival five days-post infection (5 dpi) of lower than 30\%, whereas the top five lines (DGRP_38, DGRP_319, DGRP_405, DGRP_822, DGRP_913) had an average survival of at least $94 \%$ at 5 dpi (Figure S2b). There was also considerable variance within lines, which can be partially explained by the fact that infections were conducted by multiple people across multiple treatment days. We therefore fitted a model to the data to account for the effects of treatment date and infector identity, and use fitted values from this model in subsequent analyses (Figure 1a).

\subsection{Identification of Candidate Genes}

After applying a $p$-value cut-off of $p<10^{-7}$, we retained 52 genetic variants, most (94.3\%) of which were single nucleotide polymorphisms (SNPs), as potentially associated with E. faecalis survival in $D$. melanogaster (Figure $1 \mathrm{~b}$ solid red line, Table S1). This $p$-value threshold results in a fairly high false discovery rate (FDR) of $23.8 \%$. Therefore, to further narrow down the list of candidate genes (GOIs) for validation, we validated only those genes associated with SNPs with $p<10^{-8}$ (which equates to a false discovery rate of $2.4 \%$, Figure $1 \mathrm{~b}$ dashed red line), with the following exceptions. First, two highly significant $\left(p<10^{-9}\right)$ SNPs were found in the intron of krishah (kri), and two additional SNPs with slightly lower significance $\left(p<10^{-7}\right)$ were found in the $5^{\prime}$ untranslated region (UTR) of kri (Figure S3). $k r i$ is tightly clustered with the genes $S 6 k$ and mad2 (Figure S3). All four SNPs are also tightly linked making it difficult to infer the causal SNP. SNPs in this region have also been previously identified as being associated with survival after Pseudomonas aeruginosa infection in the DGRP [13]. We therefore included all three of these genes as candidates for RNAi knockdown of gene expression. Second, one highly significant SNP (2L_17921046) occurred in a genomic region devoid of genes, and so could not be validated further. Third, we did not test the role of two genes associated with highly significant SNPs (Ncc69 and Glc-AT-P). In total, we identified six candidate genes (hereafter genes of interest, GOI) and four neighbor genes as targets for further analysis via RNAi knockdown (Table S2). For the genomically clustered GOIs $S 6 k$, mad2 and kri, a single neighbor gene (CG42272) was used. 

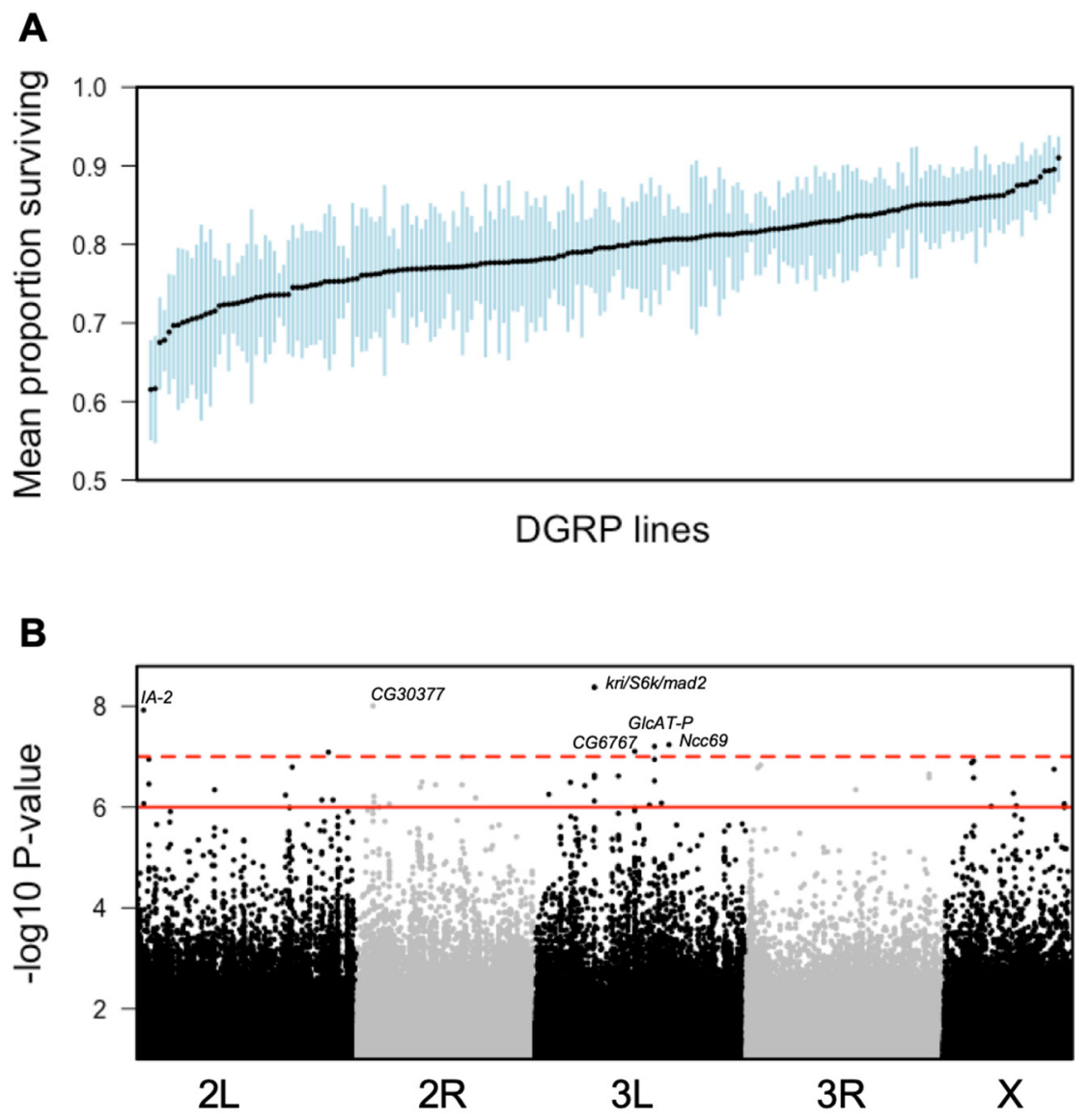

Figure 1. Genome-wide association study (GWAS) analysis using fitted data. (A) Distribution of survival rates amongst DGRP lines infected with $E$. faecalis after fitting a model to control for the effects of Date, Infector and Wolbachia status. Black line represents fitted average for a model of survival taking into account the fixed effects of infector and date of infection. The plot is sorted by increasing survival, and blue bars indicate standard error of the mean, per line. (B) Manhattan plot of genetic variants across the D. melanogaster genome and their association with E. faecalis survival. $p$-value is plotted as $-\log _{10} p$-value. Solid red line indicates $p$-value cut-off of $10^{-7}$ (which equates to a $-\log _{10} p$-value of 6). Points above this line were used for generating list of variants putatively associated with survival after infection (see Table S1). Dashed red line indicates $p$-value cut-off of $10^{-8}$ (which equates to a $-\log _{10} p$-value of 7). Most variants above this line were selected for candidate gene analysis; variants associated with genes are labelled with gene name. Black and grey shading delineates chromosomes.

\subsection{Candidate Gene Analyses}

Of the six GOIs, we found significant associations between $E$. faecalis infection and survival with two genes. There was a marginally significant trend for lower survival after infection in kri-flies $(n=111$ GOI and 177 control, Line effect $p=0.049$, Table 1, Figure 2a). For S6k-flies, we noted a non-significant trend towards higher survival in the knockdown lines $(n=155 \mathrm{GOI}$ and 172 control, Line effect $p=$ 0.196, Table 1, Figure 2b). However, due to high overall survival in this experiment, it was difficult to determine whether knockdown of $S 6 k$ improved survival (Figure $2 b$ ). Therefore, we performed a second round of infections with a higher $E$. faecalis dose $(\mathrm{OD}=2.4)$. We indeed found higher survival of $S 6 k$-flies when infection burden was increased ( $n=142$ GOI and 150 control, Line effect $p=0.029$, Table 1, Figure 2c). kri and S6k are clustered on chromosome 3L (Figure S3), suggesting that this region may be associated with the response to E. faecalis. However, we did not find any association between infection response and knockdown of two other genes in this region-mad2 $(n=302 \mathrm{GOI}$ and 278 control, Line effect $p=0.136$, Table 1, Figure S4a) and CG42272 ( $n=100$ neighbor and 133 control, Line effect $p=0.658$, Table 1, Figure S4b). Knocking down expression of the other three GOIs, located in 
different regions of the $D$. melanogaster genome, had no significant effect on survival after E. faecalis infected ( $p>0.05$, Table 1, Figure S4 left panel). Likewise, knockdown on neighbor genes had no effect on E. faecalis infection survival ( $p>0.05$, Table 1, Figure S4 right panel).

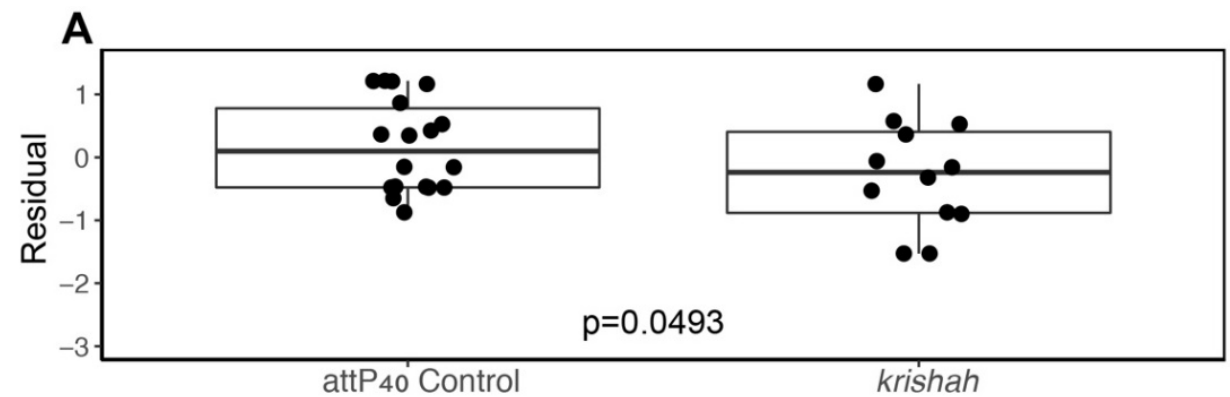

B
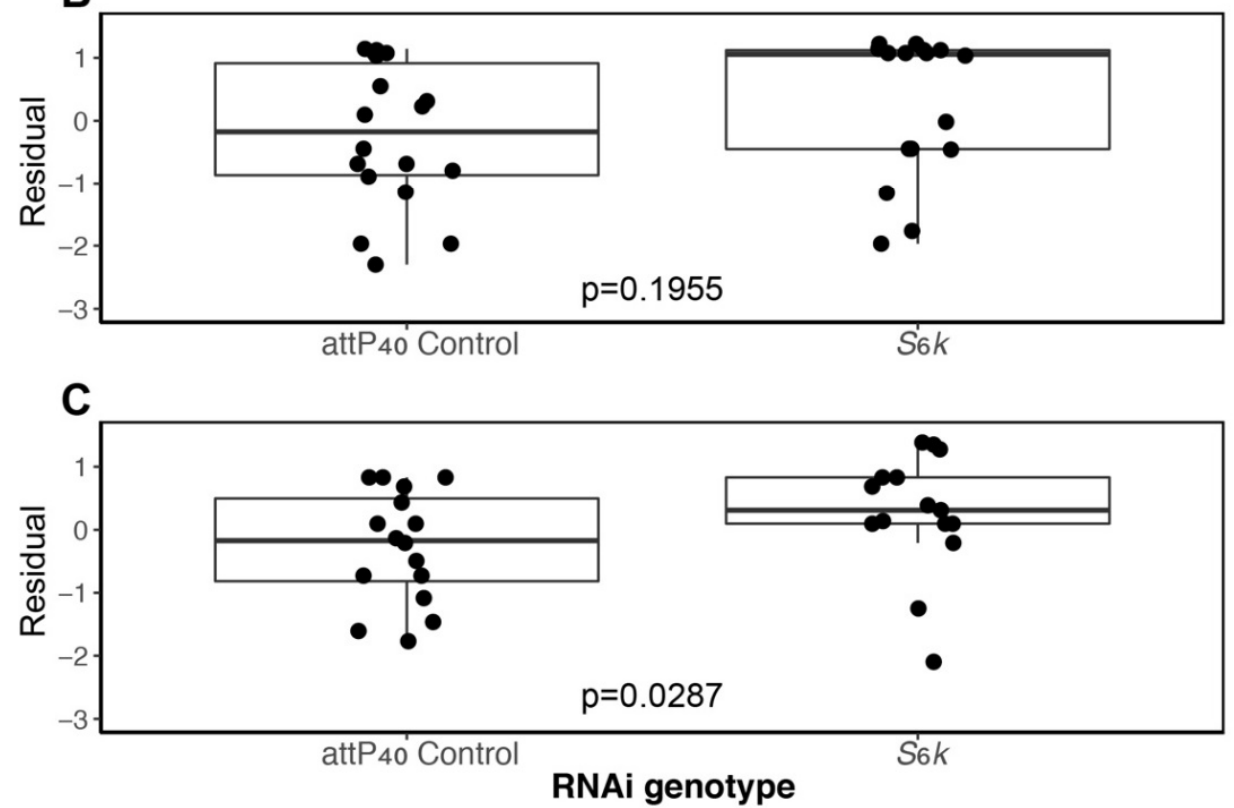

Figure 2. Survival of GOI lines versus empty cassette controls (attP40 control line 36304). (A) krishah ( $k r i$, Line 62238) infected with E. faecalis at a dose of OD = 1.5; (B) S6 kinase (S6k, Line 42572) infected with E. faecalis at a dose of $\mathrm{OD}=1.5$; (C) S6 kinase (S6k, Line 42572) infected with E. faecalis at a dose of $\mathrm{OD}=2.4$. In all cases, infections were conducted across multiple days. As such, the $y$ axis is the residuals from a model controlling for Date effects.

Table 1. Results of generalized linear models with a binomial distribution and a logit link fitted to survival data after E. faecalis (EF) infection. Genes of Interest (GOI) are listed above their genomic neighbor comparison. DF is degrees of freedom. $p$ values less than 0.05 are shown in bold.

\begin{tabular}{cccccc}
\hline Gene & Type & Factor & Deviance & Df & $\boldsymbol{p}$-Value \\
\hline Krishah & GOI & Line & 3.8655 & 1 & $\mathbf{0 . 0 4 9 3}$ \\
& & Date & 14.7613 & 3 & $\mathbf{0 . 0 0 2 0}$ \\
\hline \multirow{2}{*}{$S 6 k^{1}$} & \multirow{2}{*}{ GOI } & Line & 1.6755 & 1 & 0.1955 \\
& & Date & 7.0270 & 4 & 0.1345 \\
\hline \multirow{2}{*}{$S 6 k^{2}$} & GOI & Line & 4.7836 & 1 & $\mathbf{0 . 0 2 8 7}$ \\
& & Date & 1.0198 & 2 & 0.6006 \\
\hline \multirow{2}{*}{ mad2 } & GOI & Line & 2.2275 & 1 & 0.1356 \\
& & Date & 5.9814 & 1 & $\mathbf{0 . 0 1 4 5}$ \\
\hline
\end{tabular}


Table 1. Cont.

\begin{tabular}{|c|c|c|c|c|c|}
\hline Gene & Type & Factor & Deviance & Df & $p$-Value \\
\hline \multirow[t]{2}{*}{ CG42272 } & neighbor & Line & 0.5070 & 1 & 0.4765 \\
\hline & & Date & 6.3657 & 2 & 0.0415 \\
\hline \multirow[t]{2}{*}{ CG6767 } & GOI & Line & 0.3116 & 1 & 0.5767 \\
\hline & & Date & 6.0510 & 3 & 0.1092 \\
\hline \multirow[t]{2}{*}{ CG6761 } & neighbor & Line & 3.1126 & 1 & 0.0777 \\
\hline & & Date & 3.7781 & 3 & 0.2864 \\
\hline \multirow[t]{2}{*}{$I A 2$} & GOI & Line & 1.5029 & 1 & 0.2202 \\
\hline & & Date & 1.9210 & 3 & 0.5890 \\
\hline \multirow[t]{2}{*}{ Star } & neighbor & Line & 3.0205 & 1 & 0.0822 \\
\hline & & Date & 0.8459 & 2 & 0.6551 \\
\hline \multirow[t]{2}{*}{ CG30377 } & GOI & Line & 0.1081 & 1 & 0.7423 \\
\hline & & Date & 0.0669 & 1 & 0.7959 \\
\hline \multirow[t]{2}{*}{$D g k$} & neighbor & Line & 0.0193 & 1 & 0.8894 \\
\hline & & Date & 2.9014 & 3 & 0.4071 \\
\hline
\end{tabular}

We have previously calculated Tajima's D for all genes in the DGRP [40]. We ranked all DGRP genes from most negative to most positive and calculated the percentile ranking for each GOI and neighbor gene (Table S2). None of the genes were ranked amongst the highest or lowest $10 \%$ of genes in terms of Tajima's D, although S6k had a rather positive Tajima's D $\left(1.001,88^{\text {th }}\right.$ percentile), suggesting it may be subject to balancing selection or other forces that promote positive Tajima's D. For the remainder of the GOIs and neighbor genes, selective forces appear to be similar to the majority of the genes in this population (Table S2).

\subsection{Role of Bomanin Genes on E. faecalis Survival}

Bomanins are a newly described group of immune peptides in Drosophila [15], and have an important role in response to Gram-positive [15,22,41] and fungal [23] infections. However, to the best of our knowledge, no survival analyses have yet been performed using E. faecalis infection of lines where expression of specific Bom genes has been knocked down. We therefore investigated the two genomic regions with clusters of Bom genes more closely.

The main Bom cluster is located on chromosome $2 \mathrm{R}$ and contains 10 of the 12 Bom genes. We noted several SNPs with $p<10^{-3}$ in the $5^{\prime}$ portion of this region, including two perfectly linked SNPs in the $3^{\prime}$ UTR of BomBc1 with $p<10^{-5}$ (Figure 3a). Because mutations in the UTR can have an effect on gene expression, we next investigated whether baseline (uninduced) BomBc1 gene expression, data from [25] was influenced by these two linked SNPs, and found that individuals possessing the minor allele tended to have lower expression than those with the major allele (Figure $3 b$ ). To investigate the role of Bom genes in response to infection with E. faecalis, we knocked down expression of four Bomanin genes (BomS1, BomBc1, BomT1 and BomS4; also known as IM1, IM23, CG43202 and CG18107 respectively). We found a significant effect for $B o m B c 1$ such that individuals not expressing this gene had lowered survival compared to controls $(n=105$ BomBc1 and 105 control, Line effect $p=0.023$, Table 2, Figure 3c). Knocking down the other three Bom genes had no significant effect on survival (Table 2). Using Tajima's D values for the DGRP calculated in Chapman et al. [40], we found that BomS1 had a Tajima's D value (1.157) in the $91^{\text {st }}$ percentile of all DGRP genes (Table S3), suggesting it may be subject to balancing selection, as has previously been shown for antimicrobial peptides, including Bomanins [40]. Tajima's D for the other three Bomanin genes were similar to DGRP genes as a whole $\left(46^{\text {th }}\right.$ to $72^{\text {nd }}$ percentiles, Table S3). 

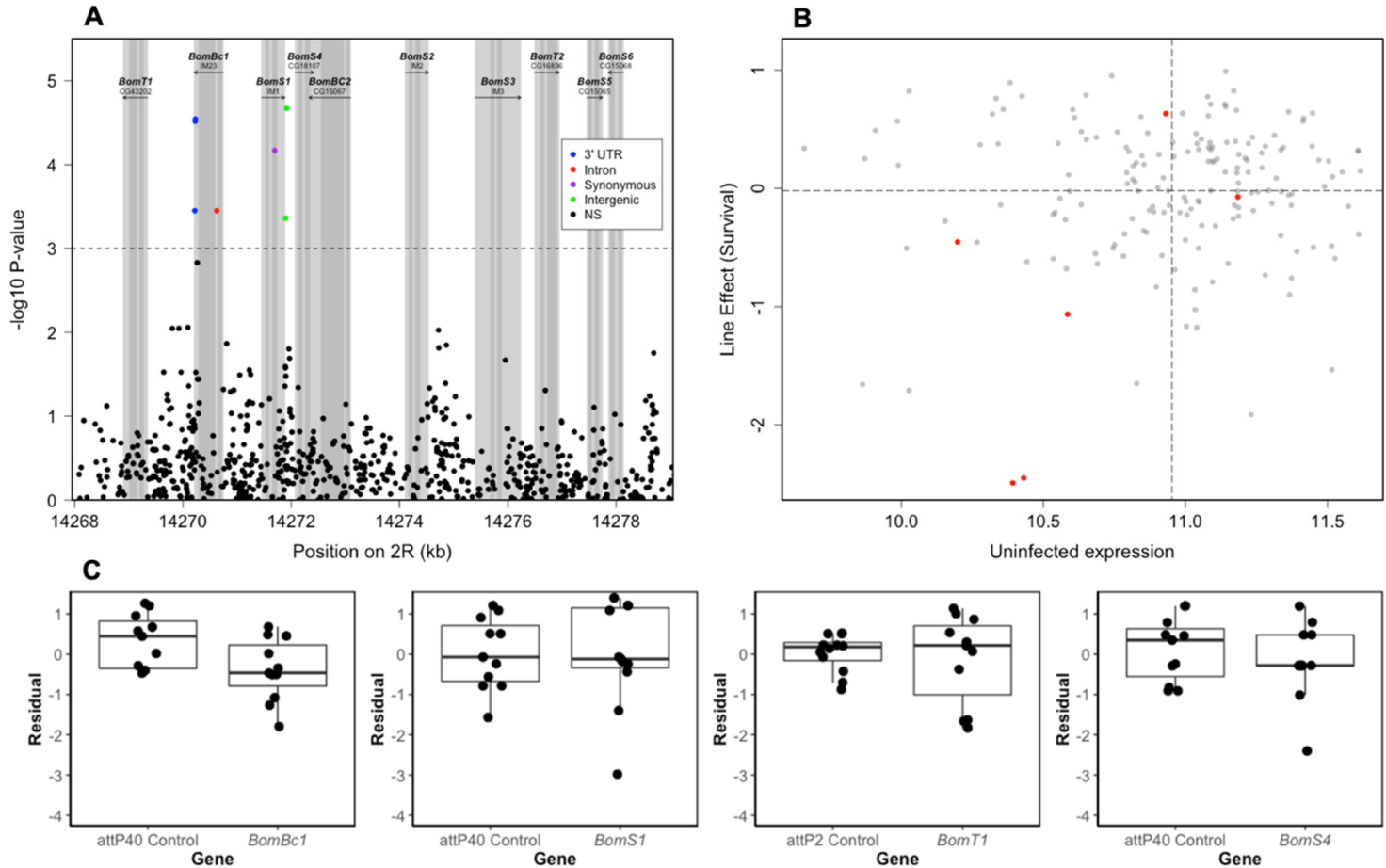

Figure 3. Relationship between Bomanin genes and E. faecalis survival. (A) Manhattan plot of genetic variants within the Bomanin cluster on chromosome $2 \mathrm{R}$ of the D. melanogaster genome and their association with $E$. faecalis survival. $p$-value is plotted as $-\log _{10} p$-value. Light grey polygons denote the limits of genes, and the dark grey polygons indicate coding sequences within genes. Color of points denotes location of variant with respect to the closest gene, as per legend. (B) Expression of the perfectly linked SNPs 2R_14270226 and 2R_14270228 in uninfected DGRP individuals plotted against survival of the same lines after infection with E. faecalis. Red dots denote the six lines homozygous for the minor allele. In general, these lines display lowered survival after E. faecalis infection (lower left quadrant). Dashed lines indicate means for survival (horizontal line) and uninfected expression (vertical line). (C) Survival of Bom-lines versus empty cassette controls after five days of infection with E. faecalis at a dose of OD = 1.5. From left to right, BomBc1, BomS1, BomT1, BomS4.

Table 2. Results of generalized linear models with a binomial distribution and a logit link fitted to survival data for Bom-lines after E. faecalis (EF) infection. Df is degrees of freedom. $p$-values less than 0.05 are shown in bold.

\begin{tabular}{ccccc}
\hline Gene & Factor & Deviance & Df & $p$-Value \\
\hline BomBc1 & Line & 5.1995 & 1 & $\mathbf{0 . 0 2 2 6}$ \\
& Date & 3.3653 & 2 & 0.1859 \\
\hline \multirow{2}{*}{ BomS1 } & Line & 0.2420 & 1 & 0.6228 \\
& Date & 9.8015 & 2 & $\mathbf{0 . 0 0 7 4}$ \\
\hline \multirow{2}{*}{ BomT1 } & Line & 0.0041 & 1 & 0.9491 \\
& Date & 10.8707 & 2 & $\mathbf{0 . 0 0 4 4}$ \\
\hline \multirow{2}{*}{ BomS4 } & Line & 0.7496 & 1 & 0.3866 \\
& Date & 2.2465 & 2 & 0.3252 \\
\hline
\end{tabular}

The other Bom cluster, comprising two Bom genes, is located on chromosome 3L. We found no evidence for SNPs associated with immune defense in this region (Figure S5), and so did not investigate these two genes further. 


\section{Discussion}

The distribution of survival proportions (Figure 1A) found in the genome wide association study indicates the presence of substantial genetic variation for defense against Enterococcus faecalis in Drosophila melanogaster even after controlling for the effects of infection date, infector identity and Wolbachia status. Since E. faecalis is present in wild populations of D. melanogaster [14], this genetic variance may have arisen due to selective pressure exerted in nature [42]. Resistance, whereby the host works to clear an infection; and tolerance, whereby the host works to limit the damage caused by infection; can both be evolutionary stable strategies for hosts, and selection will promote whichever strategy (or combination of strategies) optimizes fitness [9,43]. However, because we simply measured survival after infection, we cannot determine whether genetic variation arose via selection on tolerance or resistance to E. faecalis in the DGRP. Furthermore, the extent of the selection pressure exerted specifically by E. faecalis as opposed to other bacterial pathogens that Drosophila encounter in the wild is an open question, which will be influenced by numerous factors including pathogen encounter rates, pathogen infectivity and pathogen virulence. It may be that selection acts largely at the level of pathogen type (e.g., viral, bacterial, fungal, macro-parasitic), or at the level of classes within these classifications (e.g., Gram-positive vs Gram-negative bacteria, DNA vs RNA viruses) [42,44]. This could explain why one of the main classes of immune response genes in Drosophila, the antimicrobial peptides, are thought to have rather broad-spectrum activities $[45,46]$ rather than targeting specific pathogens [22,47]. It has recently been shown that infection with E. faecalis elicits lower AMP induction than other pathogens in Drosophila when infected at low dose [31]. Beyond broad-spectrum protective immune genes however, it may be that other genes have evolved to respond to specific pathogen threats.

The genetic variants we identified via GWAS as significantly associated with E. faecalis survival were SNPs associated with six genes, none of which were associated with canonical immune genes or GO terms related to immunity. Islet antigen 2 (IA-2) is part of the protein tyrosine phosphatase superfamily [48], and has been found to be involved in gut development during metamorphosis via modulation of insulin and hexokinase expression [49]. The $3^{\prime}$ end of the IA-2 protein contains a conserved PTP_DSP_cys protein domain characterized by a CxxxxxR catalytic loop. The PTP superfamily of proteins is part of the tyrosine phosphorylation/dephosphorylation regulatory mechanism and help cells respond to physiological changes in their environment [50]. CG30377 has been implicated in copper ion homeostasis [51]. It contains no known conserved protein domains. CG6767 is a kinase involved in purine and pyrimidine base metabolism [52], is required for glial cell differentiation [53] and affects olfactory behavior [54]. The CG6767 protein is largely comprised of a conserved $\operatorname{Prs} A$ domain. This protein superfamily is involved in nucleotide and amino acid transport and metabolism [50]. mad2 is a mitotic spindle assembly checkpoint protein, expressed in mitotic cells and important for normal cell division processes [55-57]. The protein contains a HORMA protein domain. This protein superfamily is thought to be involved in recognizing aberrant chromatin states that arise due to DNA double stranded breaks, DNA adducts and non-attachment to the mitotic spindle [50]. krishah (kri) encodes an enzyme that regulates larval growth, pre-pupal and pupal viability, and adult longevity and is homologous to human uracil phosphoribosyltransferase [58]. The Kri protein contains a UPRTase domain which belongs to the PRTases_typeI superfamily of protein domains. Type I PRTases catalyze the displacement of $\alpha-1^{\prime}$-pyrophosphate of 5-phosphoribosyl-alpha1-pyrpphosphate (PRPP) by a nitrogen-containing nucleophile [50]. S6 kinase (S6k) is a ribosomal protein kinase [59] that acts as an effector of the TORC1 growth-regulatory complex [60] and references therein] and appears to regulate various brain activities, such as synapse development [61], behavioral responses to hunger [62] and ethanol induced sedation [63].The S6k protein is largely comprised of the conserved protein domain $S T K c_{\_} p 70 s 6 \mathrm{~K}$ which belongs to the PKc_like superfamily of catalytic domains which catalyze transfer of a $\gamma$-phosphoryl group from ATP to hydroxyl groups in serine, threonine, or tyrosine residues of proteins [50]. We used the STRING database [64] to investigate known protein interactions for each of these six genes. Several genes in the $S 6 k$ network are involved in Target of Rapamycin (TOR) signaling (Figure S6). Rapamycin can act as an immunosuppressant in Drosophila, and has antifungal 
properties [65]. Downregulation of the TOR pathway has been shown to improve immune defense in female D. melanogaster [66]. We did not identify any of our other candidate genes as belonging to protein networks involving known immunity genes (Figure S6).

However, while none of the six candidate genes have previously been identified as having canonical immune functions, there is some evidence that a subset of them may have immune-related roles. First, CG30377 is somewhat induced (up to 3-fold) by Escherichia coli in S2 cells [67]. Second, a separate GWAS identified two SNPs associated with the clustered genes kri, S6k and mad2 as significantly associated with Pseudomonas aeruginosa defense in the DGRP [13]. Both SNPs were synonymous substitutions in kri and therefore occurred upstream of $S 6 k$ and downstream of mad2. Interestingly, both E. coli and P. aeruginosa are Gram-negative bacteria, whereas E. faecalis is Gram-positive. In Drosophila, bacterial pathogens are controlled by two different innate immune pathways. The immune deficiency (IMD) pathway is generally triggered by Gram-negative bacteria, whereas the Toll pathway is generally triggered by Gram-positive bacteria and fungi $[68,69]$. The fact that our GWAS with a Gram-positive bacterium identified a subset of genes that had previously been identified as responding to Gram-negative bacteria suggests these genes may be stimulated upstream of the Toll and IMD pathways or through cross-talk between these pathways and others. In particular, IMD activation downregulates the TOR pathway [70], and TOR suppression improves immunity in Drosophila [66]. Given the role of $S 6 k$ as an effector of TOR signaling (Figure S6), this may explain why this gene has been previously identified as playing a role in defense against Gram-negative infections. In mammals, mTOR has complex interactions with Toll-like receptor innate immune responses [71], which may help explain the role of $S 6 k$ in mediating survival against a Gram-positive bacterium in Drosophila. Consistent with this, we found that reducing the expression of $S 6 k$ improves survival against E. faecalis, suggesting this may be mediated via downregulation of the TOR pathway or an intermediary.

Validation by RNAi knockdown confirmed only two of the six candidate genes as having a significant association with E. faecalis survival when knocked down. S6k appears to be a negative regulator of $D$. melanogaster defense against $E$. faecalis infection, such that knocking down expression of this gene improves survival prospects for the fly. In contrast, krishah appears to be a positive regulator of the D. melanogaster defense against E. faecalis infection, such that knocking down expression of this gene reduces fly survival.

It is important to note that our GWAS was conducted with a single set of conditions (i.e., males infected with a specific E. faecalis dose and maintained on cornmeal-molasses-yeast food at $22{ }^{\circ} \mathrm{C}$ on a 12-h day/night cycle). A different set of conditions may have yielded different significant GWAS hits. For example, we have previously shown that dietary composition affects immune defense [12]. Furthermore, other factors, such as genome size, could influence identification of significant GWAS hits. Genome size can affect phenotypic traits such as survival to adulthood in the DGRP, apparently via gene expression changes that affect metabolism [72]. It is noteworthy that lines with large genome sizes upregulate genes involved in TOR signaling [72], which could have implications for immunity, as discussed above.

Bomanins are a group of AMP-like genes that appear to be key effectors of the Drosophila immune response to Gram-positive infections $[15,22,23]$. As such, we anticipated that Bomanin genes may be involved in response to E. faecalis infection in the DGRP. While we found some evidence for associated SNPs in the Bom region (Figure 3A), and that uninfected expression of the two (perfectly linked) SNPs in the 3'UTR of BomBc1 were potentially associated with defense amongst DGRP lines (Figure 3B), our RNAi candidate gene results were equivocal. BomBc1-mutants had significantly lower survival. However, we found no associations with the other three genes we tested (Figure 3C). It is possible that Bomanins have some functional redundancy, such that no one Bomanin protein is essential for controlling a specific infection, instead a cocktail of Bom proteins may help to bring the infection under control. Indeed, Clemmons et al. [15], Hanson et al. [22] and Lin et al. [41] have all found that mutant flies (Bom ${ }^{\Delta 55 C}$ ) lacking ten of the twelve Bom genes have extremely low survival when infected with E. faecalis, whereas our knockdown of individual Bom genes generally had little effect 
on survival, with the exception of BomBc1. Synergistic effects of AMPs have previously been shown in-vitro, e.g., [73-75] and in-vivo e.g., [76]. Additionally, it has recently been proposed that short-form Bomanins are particularly important for the Drosophila response to Candida infection, whereas bicipital Bomanins (e.g., BomBc1) may respond to other pathogens such as Gram-positive bacteria [41].

This work, dissecting the relationship between genetic variation and E. faecalis survival in the DGRP, improves our understanding of immunity related phenotype-genotype interactions in Drosophila. Such knowledge also elucidates how host-pathogen interactions shape the evolutionary trajectory of host populations.

Supplementary Materials: The following are available online at http://www.mdpi.com/2073-4425/11/2/234/s1, Figure S1: GWAS analysis data, Figure S2: Manhattan plot of genetic variants in an $18 \mathrm{~kb}$ window on chromosome 3L, Figure S3: Survival of GOIs (left panel) or neighbor genes (right panel) compared to empty cassette controls, Figure S4: Manhattan plot of genetic variants within the Bomanin cluster on chromosome 3L, Table S1: List of genetic variants with significant GWAS hits $\left(p<10^{-7}\right)$, Table S2: List of candidate genes and controls and Table S3: List of Bomanin lines used in this study.

Author Contributions: Conceptualization, J.R.C. and R.L.U.; methodology, J.R.C., M.A.D., R.C. and R.L.U.; formal analysis, J.R.C. and R.L.U.; data curation, J.R.C. and R.L.U.; writing-original draft preparation, J.R.C.; writing-review and editing, J.R.C., M.A.D., R.C. and R.L.U.; visualization, J.R.C. and R.L.U.; supervision, J.R.C. and R.L.U.; project administration, R.L.U.; funding acquisition, J.R.C. and R.L.U. All authors have read and agreed to the published version of the manuscript.

Funding: This research was funded by the National Institutes of Health, grant number R00 GM114714, and R01 AI139154 to R.L.U., and a subaward from P20 GM103418 to J.R.C.

Acknowledgments: We would like to thank Brian Lazzaro for providing the E. faecalis isolate. Brittny Smith helped to maintain DGRP lines in the lab. Stocks obtained from the Bloomington Drosophila Stock Center (NIH P40OD018537) were used in this study.

Conflicts of Interest: The authors declare no conflict of interest.

\section{References}

1. Hochachka, W.M.; Dhondt, A.A. Density-dependent decline of host abundance resulting from a new infectious disease. Proc. Natl. Acad. Sci. USA 2000, 97, 5303-5306. [CrossRef] [PubMed]

2. Daszak, P.; Cunningham, A.A.; Hyatt, A.D. Infectious disease and amphibian population declines. Divers. Distrib. 2003, 9, 141-150. [CrossRef]

3. Bonneaud, C.; Mazuc, J.; Gonzalez, G.; Haussy, C.; Chastel, O.; Faivre, B.; Sorci, G. Assessing the cost of mounting an immune response. Am. Nat. 2003, 161, 367-379. [CrossRef] [PubMed]

4. Hanssen, S.A.; Hasselquist, D.; Folstad, I.; Erikstad, K.E. Costs of immunity: Immune responsiveness reduces survival in a vertebrate. Proc. R. Soc. Lond. B Biol. Sci. 2004, 271, 925-930. [CrossRef]

5. Minchella, D.J. Host life-history variation in response to parasitism. Parasitology 1985, 90, 205-216. [CrossRef]

6. Hamilton, W.D. Pathogens as causes of genetic diversity in their host populations. In Population Biology of Infectious Diseases; Anderson, R.M., May, R.M., Eds.; Springer: Berlin, Germany, 1982; pp. 269-296.

7. Laine, A.-L.; Tellier, A. Heterogeneous selection promotes maintenance of polymorphism in host-parasite interactions. Oikos 2008, 117, 1281-1288. [CrossRef]

8. Hirschhorn, J.N.; Daly, M.J. Genome-wide association studies for common diseases and complex traits. Nat. Rev. Genet. 2005, 6, 95-108. [CrossRef]

9. Howick, V.M.; Lazzaro, B.P. The genetic architecture of defence as resistance to and tolerance of bacterial infection in Drosophila melanogaster. Mol. Ecol. 2017, 26, 1533-1546. [CrossRef]

10. Magwire, M.M.; Fabian, D.K.; Schweyen, H.; Cao, C.; Longdon, B.; Bayer, F.; Jiggins, F.M. Genome-wide association studies reveal a simple genetic basis of resistance to naturally coevolving viruses in Drosophila melanogaster. PLoS Genet. 2012, 8, e1003057. [CrossRef]

11. Palmer, W.H.; Medd, N.C.; Beard, P.M.; Obbard, D.J. Isolation of a natural DNA virus of Drosophila melanogaster, and characterisation of host resistance and immune responses. PLoS Pathog. 2018, 14, e1007050. [CrossRef]

12. Unckless, R.L.; Rottschaefer, S.M.; Lazzaro, B.P. The complex contributions of genetics and nutrition to immunity in Drosophila melanogaster. PLoS Genet. 2015, 11, e1005030. [CrossRef] [PubMed] 
13. Wang, J.B.; Lu, H.-L.; St Leger, R.J. The genetic basis for variation in resistance to infection in the Drosophila melanogaster genetic reference panel. PLoS Pathog. 2017, 13, e1006260. [CrossRef] [PubMed]

14. Cox, C.R.; Gilmore, M.S. Native microbial colonization of Drosophila melanogaster and its use as a model of Enterococcus faecalis pathogenesis. Infect. Immun. 2007, 75, 1565-1576. [CrossRef] [PubMed]

15. Clemmons, A.W.; Lindsay, S.A.; Wasserman, S.A. An effector peptide family required for Drosophila Toll-mediated immunity. PLoS Pathog. 2015, 11, e1004876. [CrossRef] [PubMed]

16. Chandler, J.A.; Lang, J.M.; Bhatnagar, S.; Eisen, J.A.; Kopp, A. Bacterial communities of diverse Drosophila species: Ecological context of a host-microbe model system. PLoS Genet. 2011, 7, e1002272. [CrossRef]

17. Lazzaro, B.P. A Population and Quantitative Genetic Analysis of the Drosophila Melanogaster Antibacterial Immune Response. Ph.D. Thesis, Pennsylvania State University, Centre County, PA, USA, 2002.

18. Lazzaro, B.P.; Sackton, T.B.; Clark, A.G. Genetic variation in Drosophila melanogaster resistance to infection: A comparison across bacteria. Genetics 2006, 174, 1539-1554. [CrossRef]

19. Duneau, D.F.; Kondolf, H.C.; Im, J.H.; Ortiz, G.A.; Chow, C.; Fox, M.A.; Eugenio, A.T.; Revah, J.; Buchon, N.; Lazzaro, B.P. The Toll pathway underlies host sexual dimorphism in resistance to both Gram-negative and Gram-positive bacteria in mated Drosophila. BMC Biol. 2017, 15, 124. [CrossRef]

20. Rutschmann, S.; Kilinc, A.; Ferrandon, D. Cutting edge: The Toll pathway is required for resistance to Gram-positive bacterial infections in Drosophila. J. Immunol. 2002, 168, 1542-1546. [CrossRef]

21. Sackton, T.B.; Lazzaro, B.P.; Clark, A.G. Genotype and gene expression associations with immune function in Drosophila. PLoS Genet. 2010, 6, e1000797. [CrossRef]

22. Hanson, M.A.; Dostálova, A.; Ceroni, C.; Poidevin, M.; Kondo, S.; Lemaitre, B. Synergy and remarkable specificity of antimicrobial peptides in vivo using a systematic knockout approach. eLife 2019, 8, e44341. [CrossRef]

23. Lindsay, S.A.; Lin, S.J.H.; Wasserman, S.A. Short-form Bomanins mediate humoral immunity in Drosophila. J. Innate. Immun. 2018, 10, 306-314. [CrossRef] [PubMed]

24. Mackay, T.F.C.; Richards, S.; Stone, E.A.; Barbadilla, A.; Ayroles, J.F.; Zhu, D.H.; Casillas, S.; Han, Y.; Magwire, M.M.; Cridland, J.M.; et al. The Drosophila melanogaster Genetic Reference Panel. Nature 2012, 482, 173-178. [CrossRef] [PubMed]

25. Huang, W.; Massouras, A.; Inoue, Y.; Peiffer, J.; Ramia, M.; Tarone, A.M.; Turlapati, L.; Zichner, T.; Zhu, D.H.; Lyman, R.F.; et al. Natural variation in genome architecture among 205 Drosophila melanogaster Genetic Reference Panel lines. Genome Res. 2014, 24, 1193-1208. [CrossRef] [PubMed]

26. Peng, J.; Zipperlen, P.; Kubli, E. Drosophila sex-peptide stimulates female innate immune system after mating via the Toll and Imd pathways. Curr. Biol. 2005, 15, 1690-1694. [CrossRef]

27. McGraw, L.A.; Gibson, G.; Clark, A.G.; Wolfner, M.F. Genes regulated by mating, sperm, or seminal proteins in mated female Drosophila melanogaster. Curr. Biol. 2004, 14, 1509-1514. [CrossRef]

28. Short, S.M.; Wolfner, M.F.; Lazzaro, B.P. Female Drosophila melanogaster suffer reduced defense against infection due to seminal fluid components. J. Insect. Physiol. 2012, 58, 1192-1201. [CrossRef]

29. Butterworth, F.M.; Emerson, L.; Rasch, E.M. Maturation and degeneration of the Fat-body in the Drosophila larva and pupa as revealed by morphometric analysis. Tissue Cell 1988, 20, 255-268. [CrossRef]

30. Felix, T.M.; Hughes, K.A.; Stone, E.A.; Drnevich, J.M.; Leips, J. Age-specific variation in immune response in Drosophila melanogaster has a genetic basis. Genetics 2012, 191, 989-1002. [CrossRef]

31. Chambers, M.C.; Jacobson, E.; Khalil, S.; Lazzaro, B.P. Consequences of chronic bacterial infection in Drosophila melanogaster. PLoS ONE 2019, 14, e0224440. [CrossRef]

32. Duneau, D.; Ferdy, J.B.; Revah, J.; Kondolf, H.; Ortiz, G.A.; Lazzaro, B.P.; Buchon, N. Stochastic variation in the initial phase of bacterial infection predicts the probability of survival in D. melanogaster. eLife 2017, 6, e28298. [CrossRef]

33. Perkins, L.A.; Holderbaum, L.; Tao, R.; Hu, Y.H.; Sopko, R.; McCall, K.; Yang-Zhou, D.; Flockhart, I.; Binari, R.; Shim, H.-S.; et al. The Transgenic RNAi Project at Harvard Medical School: Resources and validation. Genetics 2015, 201, 843-852. [CrossRef] [PubMed]

34. Abel, T.; Bhatt, R.; Maniatis, T. A Drosophila CREB/ATF transcriptional activator binds to both fat body- and liver-specific regulatory elements. Gene. Dev. 1992, 6, 466-480. [CrossRef] [PubMed]

35. Meister, M.; Lemaitre, B.; Hoffmann, J.A. Antimicrobial peptide defense in Drosophila. Bioessays 1997, 19, 1019-1026. [CrossRef] [PubMed] 
36. R Core Team. R: A Language and Environment for Statistical Computing; R Foundation for Statistical Computing: Vienna, Austria, 2017.

37. Bates, D.; Machler, M.; Bolker, B.M.; Walker, S.C. Fitting linear mixed-effects models using lme4. J. Stat. Softw. 2015, 67, 1-48. [CrossRef]

38. Wickham, H. ggplot2: Elegant Graphics for Data Analysis; Springer: New York, NY, USA, 2016.

39. Wei, T.; Simko, V. R Package "Corrplot": Visualization of a Correlation Matrix. 2017. Available online: https://github.com/taiyun/corrplot (accessed on 11 November 2019).

40. Chapman, J.R.; Hill, T.; Unckless, R.L. Balancing selection drives the maintenance of genetic variation in Drosophila antimicrobial peptides. Genome Biol. Evol. 2019, 11, 2691-2701. [CrossRef]

41. Lin, S.J.H.; Fulzele, A.; Cohen, L.B.; Bennett, J.; Wasserman, S.A. Bombardier enables delivery of short-form Bomanins in the Drosophila Toll response. Front. Immunol. 2020, 10, 3040. [CrossRef]

42. Duxbury, E.M.L.; Day, J.P.; Vespasiani, D.M.; Thüringer, Y.; Tolosana, I.; Smith, S.C.L.; Tagliaferri, L.; Kamacioglu, A.; Lindsley, I.; Love, L.; et al. Host-pathogen coevolution increases genetic variation in susceptibility to infection. eLife 2019, 8. [CrossRef]

43. Schmid-Hempel, P. Evolutionary Parasitology; Oxford University Press: Oxford, UK, 2011; p. 516.

44. Hill, T.; Koseva, B.S.; Unckless, R.L. The genome of Drosophila innubila reveals lineage-specific patterns of selection in immune genes. Mol. Biol. Evol. 2019, 36, 1405-1417. [CrossRef]

45. Bulet, P.; Stocklin, R. Insect antimicrobial peptides: Structures, properties and gene regulation. Protein Peptide Lett. 2005, 12, 3-11. [CrossRef]

46. Hanson, M.A.; Lemaitre, B. New insights on Drosophila antimicrobial peptide function in host defense and beyond. Curr. Opin. Immunol. 2020, 62, 22-30. [CrossRef]

47. Unckless, R.L.; Howick, V.M.; Lazzaro, B.P. Convergent balancing selection on an antimicrobial peptide in Drosophila. Curr. Biol. 2016, 26, 257-262. [CrossRef] [PubMed]

48. Cai, T.; Krause, M.W.; Odenwald, W.F.; Toyama, R.; Notkins, A.L. The IA-2 gene family: Homologs in Caenorhabditis elegans, Drosophila and zebrafish. Diabetologia 2001, 44, 81-88. [CrossRef] [PubMed]

49. Kim, J.; Bang, H.; Ko, S.; Jung, I.; Hong, H.; Kim-Ha, J. Drosophila ia2 modulates secretion of insulin-like peptide. Comp. Biochem. Physiol. A Mol. Integr. Physiol. 2008, 151, 180-184. [CrossRef] [PubMed]

50. Lu, S.; Wang, J.; Chitsaz, F.; Derbyshire, M.K.; Geer, R.C.; Gonzales, N.R.; Gwadz, M.; Hurwitz, D.I.; Marchler, G.H.; Song, J.S.; et al. CDD/SPARCLE: The conserved domain database in 2020. Nucleic Acids Res. 2020, 48, D265-D268. [CrossRef] [PubMed]

51. Norgate, M.; Southon, A.; Zou, S.G.; Zhan, M.; Sun, Y.; Batterham, P.; Camakaris, J. Copper homeostasis gene discovery in Drosophila melanogaster. Biometals 2007, 20, 683-697. [CrossRef] [PubMed]

52. Cao, W.; Song, H.J.; Gangi, T.; Kelkar, A.; Antani, I.; Garza, D.; Konsolaki, M. Identification of novel genes that modify phenotypes induced by Alzheimer's $\beta$-amyloid overexpression in Drosophila. Genetics 2008, 178, 1457-1471. [CrossRef] [PubMed]

53. Egger, B.; Leemans, R.; Loop, T.; Kammermeier, L.; Fan, Y.; Radimerski, T.; Strahm, M.C.; Certa, U.; Reichert, H. Gliogenesis in Drosophila: Genome-wide analysis of downstream genes of glial cells missing in the embryonic nervous system. Development 2002, 129, 3295-3309.

54. Brown, E.B.; Rayens, E.; Rollmann, S.M. The gene CG6767 afects olfactory behavior in Drosophila melanogaster. Behav. Genet. 2019, 49, 317-326. [CrossRef]

55. Lince-Faria, M.; Maffini, S.; Orr, B.; Ding, Y.; Florindo, C.; Sunkel, C.E.; Tavares, A.; Johansen, J.; Johansen, K.M.; Maiato, H. Spatiotemporal control of mitosis by the conserved spindle matrix protein Megator. J. Cell Biol. 2009, 184, 647-657. [CrossRef]

56. Logarinho, E.; Bousbaa, H.; Dias, J.M.; Lopes, C.; Amorim, I.; Antunes-Martins, A.; Sunkel, C.E. Different spindle checkpoint proteins monitor microtubule attachment and tension at kinetochores in Drosophila cells. J. Cell Sci. 2004, 117, 1757-1771. [CrossRef]

57. Raich, N.; Mahmoudi, S.; Emre, D.; Karess, R.E. Mad1 influences interphase nucleoplasm organization and chromatin regulation in Drosophila. Open Biol. 2018, 8. [CrossRef] [PubMed]

58. Ghosh, A.C.; Shimell, M.; Leof, E.R.; Haley, M.J.; O'Connor, M.B. UPRT, a suicide-gene therapy candidate in higher eukaryotes, is required for Drosophila larval growth and normal adult lifespan. Sci. Rep. 2015, 5, 13176. [CrossRef] [PubMed] 
59. Watson, K.L.; Chou, M.M.; Blenis, J.; Gelbart, W.M.; Erikson, R.L. A Drosophila gene structurally and functionally homologous to the mammalian 70-kDa S6 kinase gene. Proc. Natl. Acad. Sci. USA 1996, 93, 13694-13698. [CrossRef] [PubMed]

60. Zahoor, M.-K.; Poidevin, M.; Lecerf, C.; Garrido, D.; Montagne, J. A Drosophila genetic screen for suppressors of S6kinase-dependent growth identifies the F-box subunit Archipelago/FBXW7. Mol. Genet. Genom. 2019, 294, 573-582. [CrossRef]

61. Cheng, L.; Locke, C.; Davis, G.W. S6 kinase localizes to the presynaptic active zone and functions with PDK1 to control synapse development. J. Cell Biol. 2011, 194, 921-935. [CrossRef]

62. Wu, Q.; Zhang, Y.; Xu, J.; Shen, P. Regulation of hunger-driven behaviors by neural ribosomal S6 kinase in Drosophila. Proc. Natl. Acad. Sci. USA 2005, 102, 13289-13294. [CrossRef]

63. Acevedo, S.F.; de Portugal, R.L.P.Y.C.; Gonzalez, D.A.; Rodan, A.R.; Rothenfluh, A. S6 Kinase reflects and regulates ethanol-induced sedation. J. Neurosci. 2015, 35, 15396-15402. [CrossRef]

64. Szklarczyk, D.; Gable, A.L.; Lyon, D.; Junge, A.; Wyder, S.; Huerta-Cepas, J.; Simonovic, M.; Doncheva, N.T.; Morris, J.H.; Bork, P.; et al. STRING v11: Protein-protein association networks with increased coverage, supporting functional discovery in genome-wide experimental datasets. Nucleic Acids Res. 2019, 47, D607-D613. [CrossRef]

65. Katewa, S.D.; Kapahi, P. Role of TOR signaling in aging and related biological processes in Drosophila melanogaster. Exp. Gerontol. 2011, 46, 382-390. [CrossRef]

66. Lee, J.E.; Rayyan, M.; Liao, A.; Edery, I.; Pletcher, S.D. Acute dietary restriction acts via TOR, PP2A, and Myc signaling to boost innate immunity in Drosophila. Cell Rep. 2017, 20, 479-490. [CrossRef]

67. Valanne, S.; Kleino, A.; Myllymäki, H.; Vuoristo, J.; Rämet, M. Iap2 is required for a sustained response in the Drosophila Imd pathway. Dev. Comp. Immunol. 2007, 31, 991-1001. [CrossRef] [PubMed]

68. De Gregorio, E.; Spellman, P.T.; Tzou, P.; Rubin, G.M.; Lemaitre, B. The Toll and Imd pathways are the major regulators of the immune response in Drosophila. EMBO J. 2002, 21, 2568-2579. [CrossRef] [PubMed]

69. Hoffmann, J.A.; Reichhart, J.-M. Drosophila innate immunity: An evolutionary perspective. Nat. Immunol. 2002, 3, 121-126. [CrossRef] [PubMed]

70. Davoodi, S.; Galenza, A.; Panteluk, A.; Deshpande, R.; Ferguson, M.; Grewa, S.; Foley, E. The Immune Deficiency Pathway regulates metabolic homeostasis in Drosophila. J. Immunol. 2019, 202, 2747-2759. [CrossRef]

71. Abdel-Nour, M.; Tsalikis, J.; Kleinman, D.; Girardin, S.E. The emerging role of mTOR signalling in antibacterial immunity. Immunol. Cell Biol. 2014, 92, 346-353. [CrossRef]

72. Ellis, L.L.; Huang, W.; Quinn, A.M.; Ahuja, A.; Alfrejd, B.; Gomez, F.E.; Hjelmen, C.E.; Moore, K.L.; Mackay, T.F.C.; Johnston, J.S.; et al. Intrapopulation genome size variation in D. melanogaster reflects life history variation and plasticity. PLoS Genet. 2014, 10, e1004522. [CrossRef]

73. Yu, G.Z.; Baeder, D.Y.; Regoes, R.R.; Rolff, J. Combination effects of antimicrobial peptides. Antimicrob. Agents Chemother. 2016, 60, 1717-1724. [CrossRef]

74. Marxer, M.; Vollenweider, V.; Schmid-Hempel, P. Insect antimicrobial peptides act synergistically to inhibit a trypanosome parasite. Philos. Trans. R. Soc. B 2016, 371. [CrossRef]

75. Chongsiriwatana, N.P.; Wetzler, M.; Barron, A.E. Functional synergy between antimicrobial peptoids and peptides against Gram-negative bacteria. Antimicrob. Agents Chemother. 2011, 55, 5399-5402. [CrossRef]

76. Zanchi, C.; Johnston, P.R.; Rolff, J. Evolution of defence cocktails: Antimicrobial peptide combinations reduce mortality and persistent infection. Mol. Ecol. 2017, 26, 5334-5343. [CrossRef]

(C) 2020 by the authors. Licensee MDPI, Basel, Switzerland. This article is an open access article distributed under the terms and conditions of the Creative Commons Attribution (CC BY) license (http://creativecommons.org/licenses/by/4.0/). 